\title{
Design and Performance Analysis of Multi-tier Heterogeneous Network through Coverage, Throughput and Energy Efficiency
}

\author{
Amna Shabbir \\ Department of Electronic \\ Engineering, N.E.D. UET, \\ Pakistan \\ aamna@neduet.edu.pk
}

\author{
H. R. Khan \\ Department of Electronic \\ Engineering, N.E.D. UET, \\ Pakistan \\ hashim@neduet.edu.pk
}

\author{
Syed Abbas Ali \\ Department of Computer \& \\ Information Systems \\ Engineering, N.E.D \\ UET, Pakistan
}

\author{
Safdar Rizvi \\ Department of Computer \\ Science, Bahria \\ University, Pakistan \\ safdar.bukc@bahria.edu.pk
}

\begin{abstract}
The unprecedented acceleration in wireless industry strongly compels wireless operators to increase their data network throughput, capacity and coverage on emergent basis. In upcoming 5G heterogeneous networks inclusion of low power nodes (LPNs) like pico cells and femto cells for increasing network's throughput, capacity and coverage are getting momentum. Addition of LPNs in such a massive level will eventually make a network populated in terms of base stations (BSs).The dense deployments of BSs will leads towards high operating expenditures (Op-Ex), capital expenditure (Cap-Ex) and most importantly high energy consumption in future generation networks. Recognizing theses networks issues this research work investigates data throughput and energy efficiency of 5G multi-tier heterogeneous network. The network is modeled using tools from stochastic geometry. Monte Carlo results confirmed that rational deployment of LPNs can contribute towards increased throughput along with better energy efficiency of overall network.
\end{abstract}

Keywords-energy efficiency; $5 G$ networks; heterogeneous networks; stochastic geometry

\section{INTRODUCTION}

The accelerating demands of faster data connectivity and explosive growth in high speed applications results in staggering contribution towards energy consumption in today's information and communication technology (ICT) sector [1]. Currently, ICT alone generates $10 \%$ of global $\mathrm{CO} 2$ foot print and within ICT sector, $0.5 \%$ is consumed by mobile networks alone. According to the survey done in $[2,3]$ wireless sector is expected to increase by $75 \%$ latest by 2020 . Many documented studies show that in near future there will be a situation where number of BS will exceed the number of mobile users [2-5]. In the coming five year mobile data traffic expects a 20 to 30 -fold increase which is expected to exceed $250-1.000$ fold by 2020 [2]. To facilitate such a massive number of mobile users, $5 \mathrm{G}$ HetNets capacity have to increase dramatically in a significant manner. Data traffic volume in $5 \mathrm{G}$ networks is expected to increase Exabyte monthly [5].
This increase in data rate requirement puts urgency on network operator side to increase data rate without compromising the cost and quality of service. While focusing on high data rate services, the energy consumption by network is increasing naturally which pose not only serious environmental concerns but also major economical burden on networks side as well. For more than a decade the primary focus in designing of wireless networks was high capacity, coverage, data rate, throughput maximization and low latency, whereas in recent years energy efficiency (EE) of wireless network has become the foremost figure of merit due to environmental, economical and operational [4, 10-12]. Hence in design of future generation $5 \mathrm{G}$ Heterogeneous Networks (HetNets), EE is one of the major performance markers. As illustrated in Figure 1, energy consumption reduction is important from three different perspective which consumer's side, operator's side and ecological side.

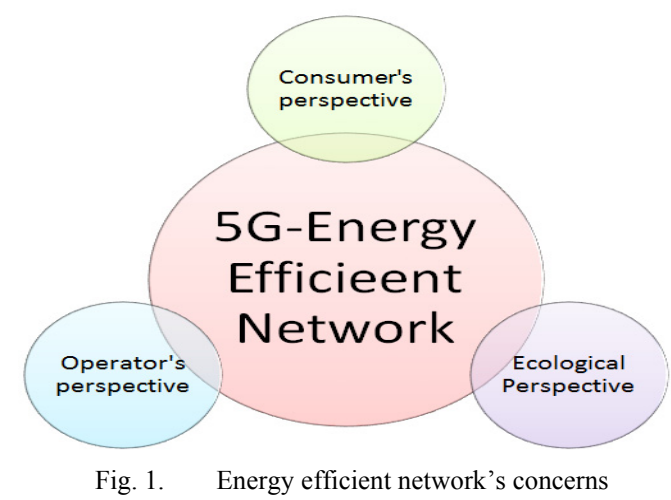

\section{A. Consumer's side Perspective}

From the perspective of mobile consumers the EE of cellular network is one of the major concern when it comes to $3 \mathrm{G}$ data services. The mobility of users becomes questionable when it comes to battery life. A recent survey done in 2016 about satisfaction of smart-phone's customers shows that iPhone received lowest marks in battery drainage [6]. The 
article [7] also emphasis on the same issue and discusses some of the potential solutions regarding wireless power transmission form energy efficient network. In a nut shell, battery life of users will be the biggest limitation for high speed or high or energy hungry devices (like 3D services, video streaming, mobile TV and multimedia), if it is not addressed quickly.

\section{B. Operators Side Perspective}

In a wireless network, the biggest contributor towards energy consumption is Macro BS, which is generally placed at the center of cell for providing basic network coverage. Usually network capacity is scaled up by increasing number of Macro BS which eventually leads to higher electricity bills due to high energy consumption. Around $18 \%$ to $20 \%$ operating expenditures (OpEx) come from BSs only. [8-11, 15]. Therefore addressing the energy crisis quickly will have significant economic benefits from operator side as well.

\section{Ecological side Perspective}

Currently ICT is solely responsible for one tenth percent of worlds total energy consumption. This percentage is continued to increase with the increasing number of cellular subscribers. Latest by 2020, three fourth sector of ICT will be wireless [12] which puts an urgency to reduce ICT related $\mathrm{CO} 2$ emissions as soon as possible. Figure 2 represents the detailed insight into the portion of energy consumed by ICT, Telecom network, cellular networks and Base stations [16]. Recognizing the importance of EE in current cellular network, this research work aims to analyze an energy aware heterogeneous network along with its data throughput.

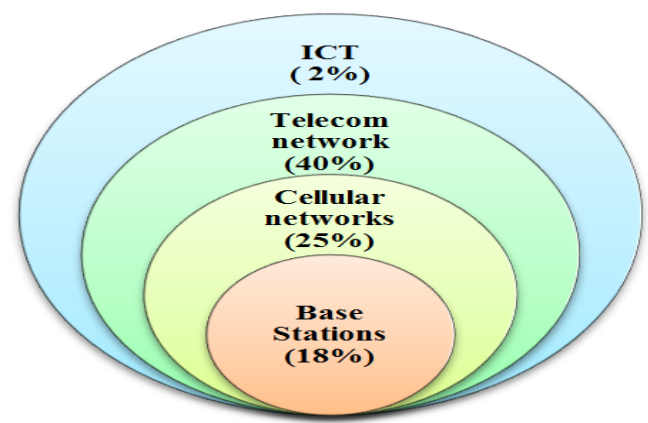

Fig. 2. The proportions of energy consumption by different subsectors

\section{MATHEMATICAL PRELIMINARIES}

A typical $5 \mathrm{G}$ HetNet consists of multiple tiers, each tier differ on the basis of its coverage, transmit power and density. Heterogeneous networks are designed and analyzed from an advance mathematical tool known as Stochastic Geometry [17, 18]. Some of the mathematical preliminaries of stochastic geometry are briefly discuss before moving into detail analysis.

\section{A. Point Process (PP)}

Random collection of points in $\mathrm{N}$-dimensional isolated points either in time or in geographical space is known as point process (PP). Mathematically, in a space of real isolated points (only integer values) point measures it is a measurable mapping
$\Phi$ from one probability space to another point measure's space. The space is usually taken as $R^{d}$ where $d>1$. Discrete sum of Dirac measure is used expressed each measure on Euclidean space E.

$$
\Phi=\sum_{i} \delta x_{i}
$$

where $x_{i}$ are the collection of random variable in Euclidian space of $\Phi$.

\section{B. Poisson Point Process (PPP)}

Poisson point process is a random point having $\lambda$ as a parameter. If $\lambda$ is constant than the PPP is called stationary PPP. XIs the average no. of PP inside a boundry region. It is not nesessary for every PPP to follow poison distribution. For real, finit $\triangle \mathrm{a}$ PP can be a PPP iff

- All points of disjoint subsets $\mathrm{S}_{1}, \ldots, \mathrm{S}_{\mathrm{n}}$, are independent;

- All random variables $\Phi(\mathrm{S})$ all sets $\mathrm{S} \in \mathrm{E}$, follows Poisson distribution.

In this paper homogeneous PPP is considered. Where homogeneous forms stationary motion invariant and density points. $\lambda$ is held to be constant throughout the space.

\section{Voronoi Tessellation}

Voronoi diagrams, voronoi tessellations are used to analyze the cellular network performance when nodes are distributed thru stochastic geometry techniques [20]. A voronoi tessellation is the distribution of a plane is such a way that each polygon contains at least one generating point. In a polygon under consideration points should be placed close to the generating point. Dirichlet tessellation is another name of Voronoi diagram. Dirichlet tessellation consists of multiple points in space $\mathrm{R}^{2}$. In this paper Voronoi tessellation on $\mathrm{R}^{\mathrm{d}}$ space and random variables are modelled through Matlab tools.

\section{Throughput}

Successful transmission of data from base station to mobile user in specific time is termed as throughput. Unit of throughput is bits per second (bps), where as in cellular network data throughput is measured typically measured in kilobits per seconds (Kbps), megabits per second (Mbps) or gigabits per second (Gbps). Sometime throughput is considered as a benchmark for measuring network performance.

\section{SYSTEM MODEL}

\section{A. Network Model}

Network considered in this paper is the multi-tier network which consist of three tiers namely Macro, Pico and Femto tiers, as shown in Figure 3. All three tiers differ in term of their densities, coverage and size. All BS are located according to PPP reflecting a heterogeneous network.

\section{B. Equations Power Consumption Model}

\section{1) Single tier network model}

Single tier network mainly consist of macro BS and MS. Macro BS consume most of the energy [15]. Around 70\% $80 \%$ of macro BS energy is consumed by power amplifiers. The power consumption at each macro BS is given as: 


$$
\mathrm{P}_{\mathrm{T}}=\mathrm{P}_{\text {static }}+\gamma \cdot \mathrm{P}_{\mathrm{tx}}
$$

$\mathrm{P}_{\mathrm{T}}$ and $\mathrm{P}_{\mathrm{tx}}$ represents the total average power consumed and transmitted power by macro BS respectively. $\mathrm{P}_{\text {static }}$ is the fixed power includes miscellaneous power consumptions due to signal processing, cooling of site etc. $\gamma$ is the scaling factor of different radiated power losses likes feeder losses. For the sake of simplicity $\gamma$ is assumed to be same throughout the network.

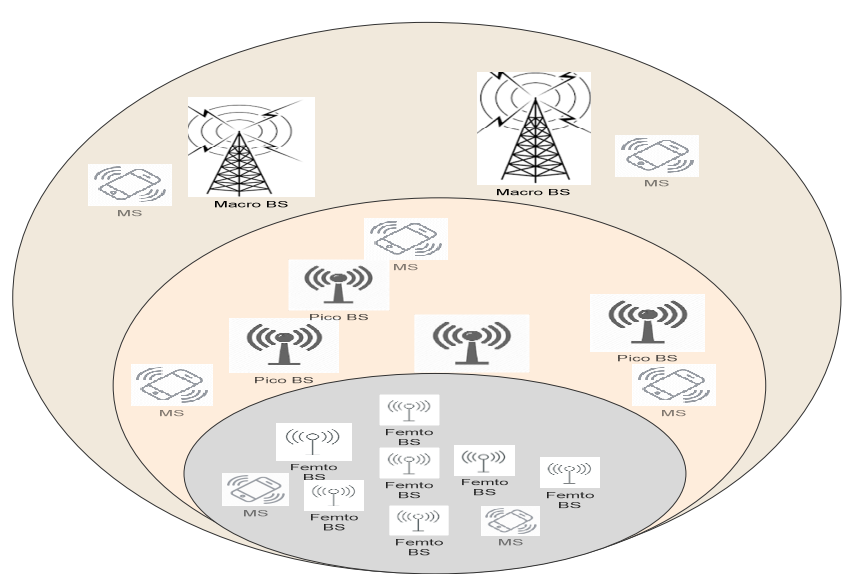

Fig. 3. A multi-tier Heterogeneous wireless network

2) Multi-tier network model

A multi-tier network is further divided in to two parts:

- 2-tier network: which consists of macro BS under laid by pico BS

- 3-tier network: which consists of macro BS under laid by pico BS and Femto BS

The BS in each tier of HetNet are distributed according to homogeneous and independent PPP $\Phi_{i}$ with density $\lambda_{i}$ and $P_{i}$ is the transmitting power transmitted of $i^{\text {th }}$ tier. BSs of every tier share same bandwidth. In $i^{\text {th }}$ any MS can connect to BS $x$ if its SINR is greater with respect to intended BS.. The threshold in $i^{\text {th }}$ tier is represented by $\beta_{i}$. Each tier can be characterized by its transmitting power, density and SINR threshold $\left\{\mathrm{P}_{\mathrm{i}}, \lambda_{\mathrm{i}}, \beta_{\mathrm{i}}\right\}$.

Mobile stations are randomly placed according PPP distribution $\Phi_{\mathrm{m}}$ with $\lambda_{\mathrm{m}}$ being its density. The channel between MS and BS is Rayleigh. BS are placed at the center with i.i.d exponential distribution. Complex channel will be considered in future work. The average received power $\mathrm{P}_{\mathrm{i}}$ of MS located at some point $\mathrm{P}_{\mathrm{i}} \in \mathrm{i}^{\text {th }}$ is:

$$
y_{\mathrm{i}}=\mathrm{P}_{\mathrm{i}} \mathrm{h}_{x_{\mathrm{i}}}\left\|x_{\mathrm{i}}\right\|^{-\alpha}
$$

Where $\alpha$ represents standard path loss exponent and ranges from 2 to 4 . In free space $\alpha=2$.

The average power consumption of the $i^{\text {th }}$ tier is given by

$$
\mathrm{P}_{\mathrm{HetNet,i}}=\lambda_{\mathrm{i}}\left(\mathrm{P}_{\text {static }, \lambda}+\gamma_{\mathrm{i}} \mathrm{P}_{\mathrm{tr}_{\mathrm{i}}}\right)
$$

Where $\lambda_{\mathrm{i}}, \mathrm{P}_{\text {static, I }}$ and $\mathrm{P}_{\mathrm{tx}}$ are is the BS density, fixed power consumption, scaling factor of different losses and output RF transmitted power in $\mathrm{i}^{\text {th }}$ tier respectively.

\section{Signal-to-interference and noise ratio (SINR)}

Assuming that MS is connected in $1^{\text {th }} \mathrm{BS}$, the expression for SINR will be given in equation 4

$$
\operatorname{SINR}_{x_{i}}=\frac{\mathrm{P}_{\mathrm{i}} \mathrm{h}_{x_{\mathrm{i}}}\left\|x_{\mathrm{i}}\right\|^{-\alpha}}{\mathrm{I}+\sigma^{2}}
$$

Where $I$ is the interference received by $x_{i}$ and $\sigma^{2}$ is AWGN power in the $i^{\text {th }}$ tier. The resulting interference is given in

$$
I=\sum_{j=1}^{M} \sum_{x \in \Phi_{i} \backslash x_{i}} \mathrm{P}_{\mathrm{i}} \mathrm{h}_{x_{\mathrm{i}}}\left\|x_{\mathrm{i}}\right\|^{-\alpha}
$$

Therefore, resulting SINR is given by

$$
\operatorname{SINR}_{x_{i}}=\frac{\mathrm{P}_{\mathrm{i}} \mathrm{h}_{x_{i}}\left\|x_{\mathrm{i}}\right\|^{-\alpha}}{\sum_{j=1}^{M} \sum_{x \in \Phi_{i} \backslash x_{i}} \mathrm{P}_{\mathrm{i}} \mathrm{h}_{x_{i}}\left\|x_{\mathrm{i}}\right\|^{-\alpha}+\sigma^{2}}
$$

Assuming $h_{x_{\mathrm{i}}} \sim \exp (1)$ for simplicity in $\tau \eta \varepsilon$ rest of the paper. MS can connect to any BS which provides strongest signal. A typical MS is said to be in coverage region if:

$$
\max \operatorname{SINR}(x)>\beta_{\mathrm{i}} \quad x \in \Phi_{i}
$$

Where $\beta_{\mathrm{i}}$ is the threshold, for simplicity it is also assume that $\beta_{\mathrm{i}}>1$, which means that in entire network at most one BS can provide SINR larger than threshold allowing any MS to connect with at most one BS at one time. For the sake of simplicity interference effect is ignored in simulated results.

\section{Energy Efficiency (EE) and Throughput}

The EE of any network is defined as the ratio of maximum achievable data rate per unit of transmitted power. Mathematically,

$$
\mathrm{EE}:=\frac{\mathrm{R}}{\mathrm{p}}
$$

Where $\mathrm{R}$ and $\mathrm{P}$ average data rate and consumed power by network respectively. EE is measured in bits/sec/watt or commonly known as bits/joule. The maximum achievable data rate commonly known as maximum throughput achievable in the network. The maximum throughput of $m^{\text {th }}$ user in a single tier can be defined in terms of Shannon capacity $C_{m}$

$$
\mathrm{C}_{\mathrm{m}}=\mathrm{B}_{\mathrm{m}} \log _{2}\left(1+\mathrm{SINR}_{\mathrm{m}}\right)
$$

Where $B_{m}$ is the assigned bandwidth of the $m^{\text {th }}$ user.

In case of multi-tier HetNet, this capacity will be scaled by factor $n_{i}$, where $n$ is the no of Pico or Femto BS in a $i^{\text {th }}$ tier

$$
\mathrm{C}_{\mathrm{m}_{\mathrm{i}}}=\mathrm{n}_{i} \mathrm{~B}_{\mathrm{m}} \log _{2}\left(1+\mathrm{SINR}_{\mathrm{m}}\right)
$$


The average EE of two tier i-e macro under laid by pico cells is given as

$$
\mathrm{EE}_{\text {two-tier }}=\frac{\mathrm{R}_{\text {macro }}+\sum_{\mathrm{k}=1}^{\mathrm{K}} \mathrm{R}_{\mathrm{P}_{\mathrm{i}, \mathrm{k}}}}{\mathrm{P}_{\text {macro }}+\sum_{\mathrm{k}=1}^{\mathrm{K}} \mathrm{P}_{\mathrm{P}_{\mathrm{i}, \mathrm{k}}}}
$$

Where $R_{\text {macro }}$ is the average throughput and $P_{\text {macro }}$ is the average consumed power by macro BS respectively. $R_{P_{i, k}}$ and $\mathrm{P}_{\mathrm{P}_{\mathrm{i}, \mathrm{k}}}$ represents the average throughput and average consumed power by $\mathrm{k}^{\text {th }}$ Pico station respectively.

Likewise, the EE of three tier network can be calculate as

$$
\mathrm{EE}_{\text {three-tier }}=\frac{\mathrm{R}_{\text {macro }}+\mathrm{R}_{\text {pico }}+\sum_{\mathrm{m}=1}^{\mathrm{M}} \mathrm{R}_{\mathrm{P}_{\mathrm{i}, \mathrm{m}}}}{\mathrm{P}_{\text {macro }}+\sum_{\mathrm{m}=1}^{\mathrm{M}} \mathrm{P}_{\mathrm{P}_{\mathrm{i}, \mathrm{m}}}}
$$

$\mathrm{R}_{\mathrm{P}_{\mathrm{i}, \mathrm{m}}}$ and $\mathrm{P}_{\mathrm{P}_{\mathrm{i}, \mathrm{m}}}$ present the average throughput and average consumed power by $\mathrm{m}^{\text {th }}$ femto station respectively.

\section{RESULTS AND ANALYSIS}

In this section coverage tessellation, throughput and EE of single, two tier and three tier network is analyzed. Data throughput and EE are explored in detail for two tier and three tier network, with special focus on how number of base station and number of tiers effect on EE and data throughput of multitier HetNet.

TABLE I. SIMULATION PARAMETERS FOR MACRO CELLS

\begin{tabular}{|c|c|}
\hline Cellular Layout & Hexagonal grid, 3 sectors per site \\
\hline Inter-site distance & $500 \mathrm{~m}$ \\
\hline UE power class & $23 \mathrm{dBm}(200 \mathrm{~mW})$ \\
\hline UE distribution & $80 \%$ inside the building \\
\hline
\end{tabular}

TABLE II. SIMULATION PARAMETERS FOR PICO CELLS

\begin{tabular}{|c|c|}
\hline Carrier frequency & $2000 \mathrm{MHz}$ \\
\hline Bandwidth & $10 \mathrm{MHz}$ \\
\hline Path loss model & $\mathrm{L}=38+30 \log 10(\mathrm{R}) ; \mathrm{R} \mathrm{in} \mathrm{m}$ \\
\hline Max Pico TX power 24dBm & $24 \mathrm{dBm}$ \\
\hline Antenna gain & $0 \mathrm{dBi}$ or $2 \mathrm{dBi}$ \\
\hline Outdoor wall penetration loss & $10 \mathrm{~dB}$ \\
\hline Pico BS noise figure & $6 \mathrm{~dB}$ \\
\hline Min separation UE to Pico BS & $2 \mathrm{~m}$ \\
\hline
\end{tabular}

TABLE III. SIMULATION PARAMETERS FOR FEMTO CELLS

\begin{tabular}{|c|c|}
\hline Max output power & $20 \mathrm{dBm}$ \\
\hline Propagation model & $\mathrm{PL}(\mathrm{dB})=127+30 \log 10(\mathrm{R} / 1000), \mathrm{R}$ in $\mathrm{m}$ \\
\hline $\begin{array}{l}\text { Log-normal shadowing } \\
\text { standard deviation }\end{array}$ & $10 \mathrm{~dB}$ \\
\hline
\end{tabular}

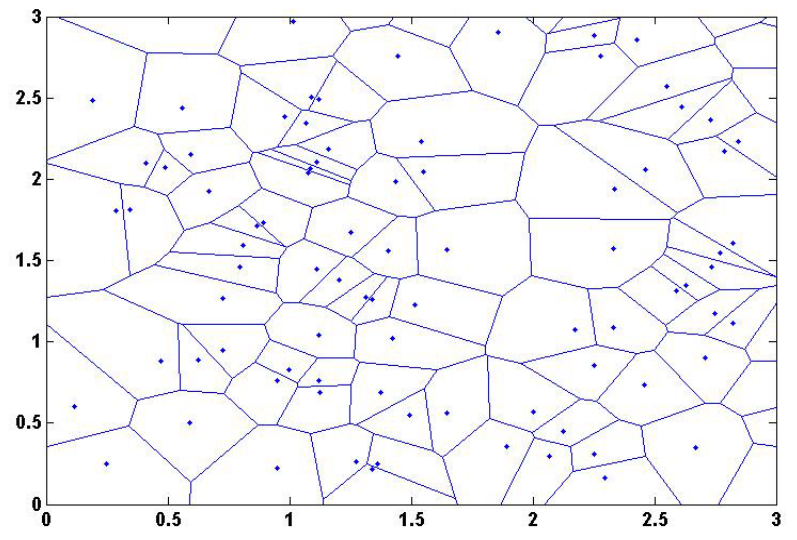

Fig. 4. Single tier network with BS distribution as PPP

The simulations parameters for network under consideration follow that in 3GPP TR 36.931 version 9 and 3GPP TR 36.922 version 10 [14-15] which are given in Table [I-III]. For single tier network, i-e macro BSs only. Coverage tessellation plot is shown in Figure 4. Macro BSs are distributed according to PPP throughout the network. Similarly, coverage tessellation for two tier network is shown in Figure 5. Figure 5 consist of two tier network Macro and Pico only. It comprises of Macro (blue dots) underlaid by pico cell (red dots) only. The BS density of pico cells are twice than macro BS. Through Monte Carlo simulation, throughput of two tier network is investigated. Macro BSs are placed homogeneous whereas Pico cells are distributed according to PPP with twice BS density of macro cells. The total BW is divided uniformly across all MS. The data throughput is calculated with known SNIR at the receiver. Figure 6 shows data throughput in Mbps, which shows that while increasing number of Pico BS gradually the throughput of two tier network is increased.

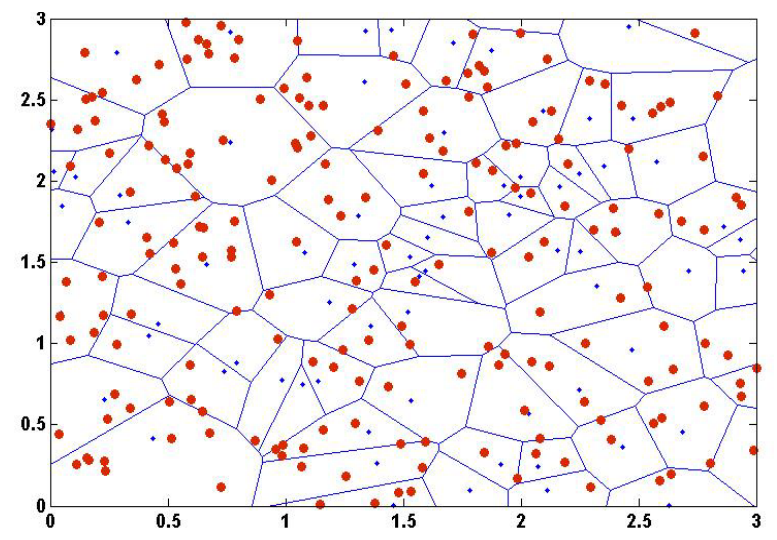

Fig. 5. Two tier network Pico (red) and Macro (blue) with $\left(\lambda_{\text {pico }}=2 \lambda_{\text {macro }}\right)$

Figure 7 shows the EE of two tier network using Equation 11. Comparison of EE in bits/joule is plotted for different number of Pico BS. Two tiers network's EE can be increased if number of Pico BS are increase reasonably e.g. 6 to 10 . However, increasing Pico base station will results in increased co-channel interference in some over lapping areas due to its smaller size and placement inside a macro cell. Nevertheless 
capacity of Hetnet will improves significantly if rational numbers of Pico station are placed. Three tier network is designed by placing Femto cells inside two tier network forming a three tier network which consist of Macro, Pico and Femto BS. Average data throughput and EE of three tier network is analyzed below. Figure 8 represents coverage tessellation plot for three tier network. Macro and pico BSs underlaid by femto BSs (green). The density of femto BSs is ten times higher than macro BSs. All tiers are modelled by PPP distribution. Figure 9 show the average throughput generated by Femto cells as a function of transmitting power. It is observed that in a three tier network when transmitting power rises from $10 \mathrm{dBm}$ to $20 \mathrm{dBm}$, there is linear increase in data throughput whereas increasing its transmitting power from $20 \mathrm{dBm}$ to $28 \mathrm{dBm}$ will result in exponential growth in data rate. For assessing EE attained by Femto cells only, keeping transmitting power constant, statistical comparison of data rate and energy in bits/joule has been done for multiple Femto BS as depicted in Figure 10. From the statistical plot it is evident that increasing the number of small cell i-e Femto cells will results in increase energy efficiency due to the reduce distance between BS and mobile user. Table IV shows the data rate and energy when femto BS increases from one to six in three tier HetNet.

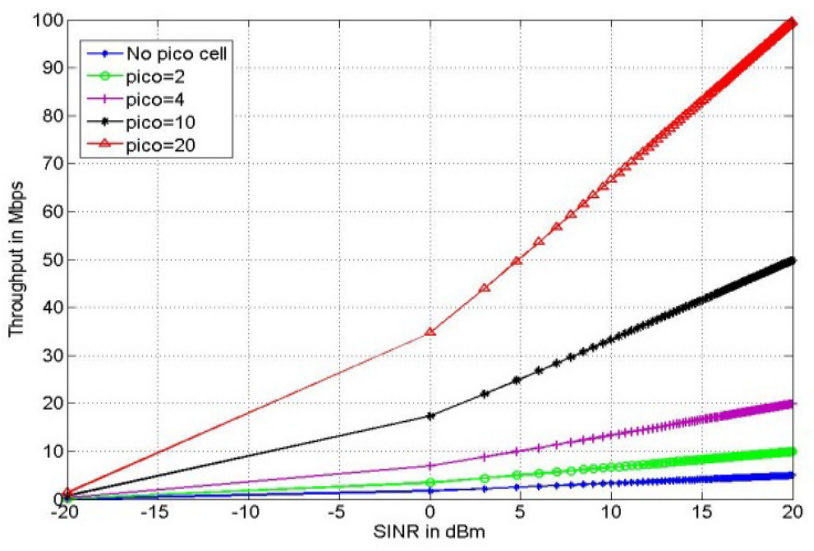

Fig. 6. Average throughput of two-tier network

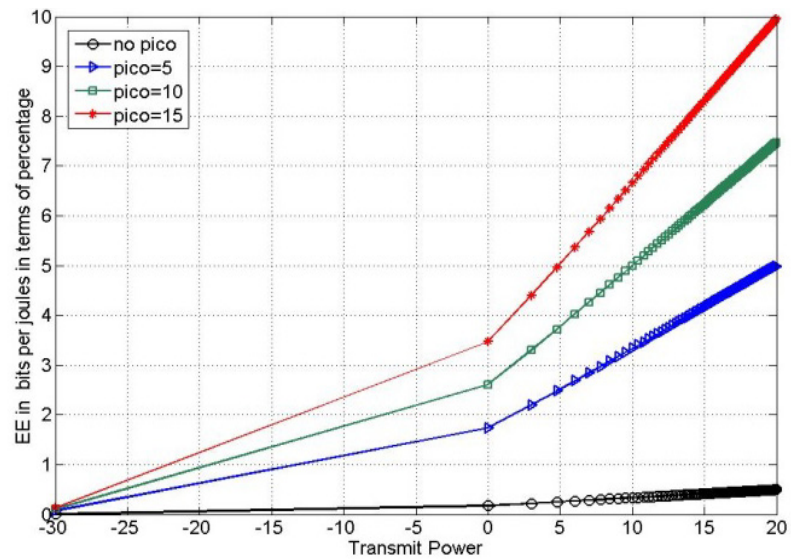

Fig. 7. Energy Efficiency of two tier network.

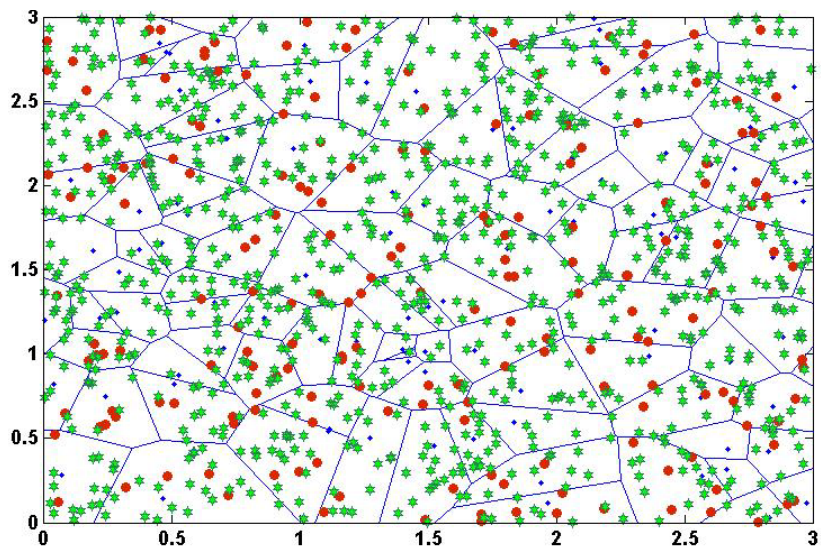

Fig. 8. Three tier network with $\left(\lambda_{\text {pico }}=2 \lambda_{\text {macro }}\right)$. Pico (red) and Macro (blue) PPP with $\left(\lambda_{\text {pico }}=2 \lambda_{\text {macro }}\right)$ and $\left(\lambda_{\text {femto }}=10 \lambda_{\text {macro }}\right)$. Femto (green), Pico (red) and Macro (blue)

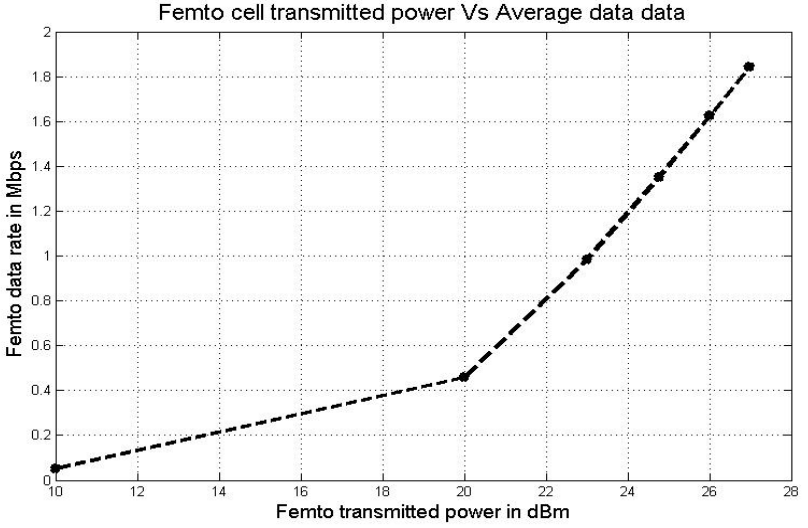

Fig. 9. Femto cells data throughput

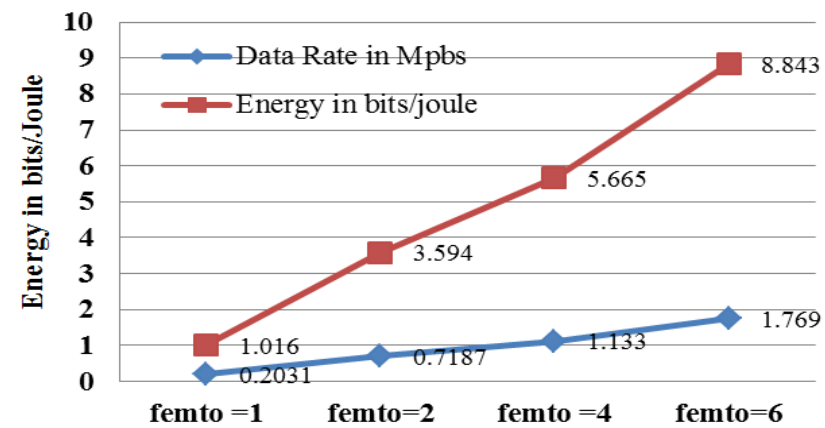

Fig. 10. Statistical comparison of data rate and energy for three tier network

TABLE IV. DATA RATE AND ENERGY COMPARISION FOR MULTIPLE FEMTO CELLS

\begin{tabular}{|c|c|c|c|c|}
\hline Parameter & Femto= & Femto=2 & Femto=4 & Femto=6 \\
\hline Data Rate in Mpbs & 0.2031 & 0.7187 & 1.133 & 1.769 \\
\hline Energy in bits/joule & 1.016 & 3.594 & 5.665 & 8.843 \\
\hline
\end{tabular}




\section{CONCLUSION}

In this research paper coverage tessellations, data throughput and energy efficiency is being investigated through deployment of multi-tier heterogeneous network. A multi- tier network is form by two different topologies namely two-tier (macro-Pico) and three-tier (macro, Pico and Femto). All networks are modeled using stochastic geometry. Coverage tessellations, data throughput and energy efficiency analyses of both networks. In particularly effect of number of Pico and Femto base stations on data throughput and on energy efficiency is explored in detail. Statistical and numerical analysis confirmed that $5 \mathrm{G}$ heterogeneous network throughput can be enhanced to greater extent if small base stations i-e Pico and Femto cell are increased in rational manner. In addition, wise deployment of optimal number of BSs can lead to higher energy efficiency. Moreover, in this research work energy efficiency investigation is only dependent on power transmitted by base stations within the tier under consideration. It is expected that energy efficiency and data throughput can further be improved if optimal power transmission and adaptive power control strategies are use.

\section{REFERENCES}

[1] D. Muirhead, M. Ali Imran, K. Arshad, "A Survey of the Challenges, Opportunities and Use of Multiple Antennas in Current and Future 5G Small Cell Base Stations", IEEE Access, vol. 4, pp. 2952-2964, 2016

[2] S. Provider, W. Papers, "Cisco Visual Networking Index: Global Mobile Data Traffic Forecast Update, 2015-2020 White Paper”, Cisco, 2016

[3] J. Zhu, "Energy Efficiency Analysis of Cellular Downlink Transmission with Network Coding over Rayleigh Fading Channels", KSII Transactions on Internet and Information Systems, Vol. 7, No. 3, pp. 446-458, 2013

[4] K. Davaslioglu, R. D. Gitlin, "5G green networking: Enabling technologies, potentials, and challenges", 2016 IEEE 17th Annual Wireless and Microwave Technology Conference (WAMICON), pp. 16, USA, 2016

[5] S. Buzzi, C. I. T. Klein, H. Poor, C. Yang, A. Zappone, "A Survey of Energy-Efficient Techniques for $5 \mathrm{G}$ Networks and Challenges Ahead", IEEE Journal on Selected Areas in Communications, Vol. 34, no. 4, pp. 697-709, 2016

[6] J. D. Power, 2016 U.S. Wireless Smartphone Satisfaction Study Volume 1,2016

[7] K. Huang, X. Zhou, "Cutting the last wires for mobile communications by microwave power transfer", IEEE Communications Magazine, Vol. 53, No. 6, pp. 86-93, 2015

[8] A. Fehske, G. Fettweis, J. Malmodin, G. Biczok, "The global footprint of mobile communications: The ecological and economic perspective", IEEE Communications Magazine, Vol. 49, No. 8, pp. 5562,2011

[9] A. Shabbir, H. R. Khan, S. A. Ali, S. Rizvi, "A Stochastic Geometrical Approach for Performance Analysis of Heterogeneous Cellular Network", International Journal of Computer Science and Information Security, Vol. 14, No. 8, pp. 467-475, 2016

[10] Research Focus Magazine, Why the EU is betting big on 5G, Digital Single Market, 2016.

[11] G. Auer, V. Giannini, C. Desset, I. Godor, P. Skillermark, M. Olsson, M. Imran, D. Sabella, M. Gonzalez, O. Blume, A. Fehske, "How much energy is needed to run a wireless network?", IEEE Wireless Communications, Vol. 18, No. 5, pp. 40-49, 2011

[12] D. Feng, C. Jiang, G. Lim, L. Cimini, G. Feng, G. Li, "A survey of energy-efficient wireless communications", IEEE Communications Surveys \& Tutorials, Vol. 15, No. 1, pp. 167-178, 2013

[13] S. Verdu, "On channel capacity per unit cost", IEEE Transactions on Information Theory, Vol. 36, No. 5, pp. 1019-1030, 1990
[14] D. Lister, "An operator's view on green radio", the Proc. IEEE Int. Workshop on Green Communications, 2009

[15] G. Rittenhouse, "Green Wireless Networks", IEEE WCNC, Paris, France, 2012

[16] M. Haenggi, Stochastic Geometry for Wireless Networks. Cambridge University Publishers, 2012

[17] M. Haenggi, J. G. Andrews, F. Baccelli, O. Dousse, M. Franceschetti. "Stochastic geometry and random graphs for the analysis and design of wireless networks", IEEE Journal on Selected Areas in Communications, Vol. 27, No. 7, 2009

[18] K. Huang, J. G. Andrews, "A stochastic-geometry approach to coverage in cellular networks with multi-cell cooperation", IEEE Global Telecommunications Conference, GLOBECOM 2011, Nepal, 5-9 Dec. 2011 